\title{
Trypanosoma cruzi: parasite antigens sequestered in heart interstitial dendritic cells are related to persisting myocarditis in benznidazole-treated mice
}

\author{
Renata Siqueira Portella, Sonia G Andrade/ ${ }^{+}$ \\ Laboratório de Chagas Experimental, Autoimunidade e Imunologia Celular, Centro de Pesquisas Gonçalo Moniz-Fiocruz, \\ Rua Valdemar Falcão 121, 40295-001 Salvador, BA, Brasil
}

We investigated whether sequestered Trypanosoma cruzi antigens found in heart interstitial dendritic cells (IDCs) contribute to the residual myocarditis found in mice following treatment with benznidazole, a specific chemotherapeutic drug. IDCs are antigen-presenting cells that are MHC-II-receptor dependent. Swiss mice were divided into two experimental groups: the 1st group was infected with the Colombian strain of $\mathrm{T}$. cruzi, which is resistant to treatment with benznidazole, and the 2nd group was infected with clone 21SF-C 3, which has a medium susceptibility to the drug. Treatment of the Colombian strain group started on the 120th day post-infection and for the 21SF-C3 strain group treatment was started on the 90th day. In both groups, treatment lasted for 90 days. The animals were sacrificed either 150 or 200 days post-treatment. The myocardium was analysed by immunohistochemistry using anti-MAC3, 33D1, CD11b and CD11c monoclonal antibodies for IDCs or anti-T. cruzi purified antibodies. Parasite antigens were expressed on the IDC membranes in both treated and untreated mice. Myocarditis subsided following treatment, evidenced by both histological and morphometrical evaluation. A reduction in the number of IDCs carrying T. cruzi antigens in the treated group indicates that the elimination of parasites influences antigen presentation with concomitant decreases in inflammation. There is a correlation between the presence of $\mathrm{T}$. cruzi antigens in these cells and the chronic focal, residual myocarditis seen in treated mice.

Key words: Trypanosoma cruzi - chronic myocarditis - heart interstitial dendritic cells - chemotherapy - benznidazole

Infection with Trypanosoma cruzi is an important cause of chronic myocarditis for people living in large areas of South and Central America where Chagas disease is endemic.

The pathogenesis of chronic Chagas cardiomyopathy is related to a delayed hypersensitivity response to the parasite invasion (Tarleton 1995, 2001, Tarleton et al. 1996, Dos Reis 1997) and is characterised by diffuse, progressive fibrotic myocarditis (Andrade 1991, 1999). However, pathogenesis is still controversial due to the paucity of parasites within the heart in chronic human cases and this has led to the hypothesis of an autoimmune origin (Cunha Neto et al. 1995, 1996, Girones \& Fresno 2003). The regression of the fibrosing myocarditis after specific chemotherapy points to the crucial role played by the parasite (Andrade et al. 1989a, b, 1991b). However, even in treated mice with negative parasitological tests, residual inflammatory infiltrates can be detected, either as isolated foci or as mild interstitial diffuse mononuclear cell infiltration (Andrade et al. 1989b). It is important to clarify the significance of these findings because the success of anti-T. cruzi treatment is related to the complete clearance of tissue lesions.

+ Corresponding author: sgandrade@bahia.fiocruz.br

Received 11 March 2009

Accepted 29 September 2009
Previous studies have demonstrated that parasite antigens that are sequestered and then expressed on the membranes of cardiac interstitial dendritic cells (IDCs) are important factors in the development of Chagas myocarditis (Andrade et al. 2000). It is important to investigate whether the antiparasitic drug-induced decrease in the parasite load can improve the healing process by interfering with antigen presentation by the IDCs and consequently reducing local inflammation. IDCs, first described by Hart and Fabre (1981), who recognised them as specialised antigen-presenting cells, have the ability to bind to antigens, to produce IL-12 (Steinman 1988, Heufler et al. 1996) and to stimulate a T lymphocyte response (Hart \& Mckenzie 1990, Flores-Romo 2001). They probably participate in the immunological process of Chagas myocarditis, considering their role in antigen presentation, their association with MHC-II products and their sensitisation of T-cells for cytokine production (Inaba et al. 1987, Steinman 1988, 1991, Drakesmith et al. 2000).

The present study attempts to correlate the presence of $T$. cruzi antigens sequestered in IDCs with the chronic focal, residual myocarditis observed in mice chronically infected with $T$. cruzi and treated with benznidazole as compared with untreated controls. Two strains of T. cru$z i$ with different degrees of susceptibility to the nitroimidazolic compound benznidazole were used: the highly resistant Colombian strain (Andrade et al. 1985) and the medium susceptibility 21SF-C3 clone (Campos et al. 2005). A histopathological, immunohistochemical and quantitative evaluation of inflammatory cells and heart 
IDCs was performed in treated and untreated mice. The participation of intracellularly sequestered parasite debris in focal lesions was also investigated

\section{MATERIALS AND METHODS}

Experimental animals - A total of 279 Swiss Webster mice weighing from $15-20 \mathrm{~g}$ raised in the animal facilities of the Centro de Pesquisas Gonçalo Moniz-Fiocruz were used. They were maintained in accordance with the ethical guidelines established by the Ethical Committee for the Use of Experimental Animals (CEUA-CPqGMFiocruz), under protocol 034.

Infection with T. cruzi - Mice were infected with two different strains of $T$. cruzi. One group was infected with the Colombian strain (T. cruzi I) (Anonymous 1999), classified as Biodeme Type III, which is resistant to treatment with benznidazole (Andrade et al. 1997); the other group was infected with clone 21SF-C3 obtained from the 21SF strain (T. cruzi II) (Anonymous 1999), classified as Biodeme Type II (Andrade et al. 1997), which is susceptible to treatment with benznidazole (Campos et al. 2005).

Inoculum - A total of $1 \times 10^{4}$ trypomastigote blood forms obtained from mice infected with each strain or clone were inoculated intraperitoneally into the animals of each experimental group.

Post-inoculation follow-up - Parasitaemia was evaluated in the acute phase of infection by microscopic examination of peripheral blood obtained from the tails of infected mice. The blood was smeared on a glass slide a cover slip was added and 50 fields at $400 \mathrm{X}$ were examined. Five animals were examined daily from the 7th-50th day of infection. Parasitaemia was expressed as the mean parasite counts obtained on each day. In the chronic phase, parasitaemia was examined every two weeks. Cumulative mortality was evaluated until the 50 th day of infection.

Experimental groups - 1st group: 177 mice were infected with the Colombian strain. A high mortality rate was measured in the acute phase ( $76.3 \%$ of the animals). The 42 survivors were followed until the chronic phase. Of those survivors, 17 were used as untreated controls and 25 were treated with benznidazole. Treatment was initiated at the 120th day of infection. 2nd group: 102 mice were infected with clone 21 SF-C3. Mortality in the acute phase was of $43 \%$. The 58 survivors were followed until the chronic phase and divided into two groups: 24 untreated controls and 34 treated with benznidazole.

Treatment - For both experimental groups, benznidazole (N-benzyl-2-nitro-1-imidazolacetamide) was administered by gavage at a dose of $100 \mathrm{mg} / \mathrm{kg} /$ day, five days per week over 90 days, starting on the 90th day of infection for the clone 21SF-C3 and on the 120th day for those infected with the Colombian strain.

Post-treatment follow-up - From 30-60 days after the end of treatment for parasitaemia, both the treated and untreated controls were evaluated. In the animals with persistently negative parasitaemia, the parasitological and serological cure tests were performed at the end of the experiment (150-200 days post-treatment). A positive result on at least one parasitological test (direct blood examination, sub-inoculation into newborn mice or haemoculture) was defined as cure failure. Mice were sacrificed for histopathological and histochemical study after anaesthesia with halothane. The mice were exsanguinated by transection of the axilar plexus. The blood was harvested with a heparinised syringe and haemoculture was performed in Warren media. Part of the blood was inoculated into suckling mice, which were daily examined for parasitemia. Non-heparinised blood samples were collected for serology.

Serological examination for specific anti-T. cruzi antibodies - Antibodies were measured by indirect immunofluorescence test (IIFT), using culture forms of $T$. cruzi as antigens and rabbit-anti-T. cruzi monospecific serum as a primary antibody at dilutions of 1:10-1:1280. For the secondary antibody, a rabbit anti-mouse $\operatorname{IgG}$ conjugated to fluorescein (Sigma) was used. The positive cut-off was 1:40. Lower titres in the IIF test were more frequently seen in the treated mice in both groups.

Cure rates - Cure was established by the evaluation of the combined results of the parasitological and serological tests in mice infected with either the Colombian strain (1st group) or clone 21SF-C3 (2nd group), both treated and untreated with benznidazole.

Histopathological study - Sacrificed mice were submitted to complete necropsies. Sections of the heart and skeletal muscles were fixed into $10 \%$ formalin and embedded in paraffin; $5 \mu \mathrm{m}$ sections were stained with haematoxylin and eosin (H\&E). The intensity of the inflammatory lesions observed on histopathological examination was expressed as: mild lesions with slight focal or diffuse mononuclear infiltrates $(+)$, moderate lesions consisting of diffuse and focal infiltrates $(++)$ and intense lesions corresponding to dense diffuse infiltrates or to focal and confluent infiltrates $(+++)$.

Immunohistochemistry: immunolabelling of T. cruzi antigens (IDCs of the heart, parasites and parasite debris) - Immunohistochemistry staining of parasite antigens, as intracellular parasites, as extracellular particulate debris or as deposits in the membranes of IDCs in the heart, was performed in $5 \mu \mathrm{m}$-thick paraffin sections after deparaffinisation and hydration. Blocking of endogenous peroxidase was done with a solution of hydrogen peroxide (3\%) diluted in methanol. After washing in distilled water and PBS, sections were treated for $15 \mathrm{~min}$ with $10 \%$ skim milk to blocking non-specific binding. Sections were treated for $30 \mathrm{~min}$ at $37^{\circ} \mathrm{C}$ with the primary antibody (a purified, specific anti-T. cruzi IgG produced in rabbits) at a dilution of 1:600 in PBS with 2\% Tween-20. After washing in PBS and 2\% Tween-20, sections were incubated in normal goat serum (Vectastain - Elite) for 20 min for additional blocking of non-specific binding. After washing in PBS, the slides were then incubated for $30 \mathrm{~min}$ at $37^{\circ} \mathrm{C}$ with the secondary antibody [goat antirabbit $\mathrm{IgG}$ - peroxidase conjugate (Sigma)] at a dilution of 1:800 in PBS. The complex was developed with $2.4 \%$ 3,3'-diaminobenzidine tetrahydrochloride (DAB) and $1 \%$ 
$\mathrm{H}_{2} \mathrm{O}_{2}$ at RT plus $1 \%$ dimethylsulfoxide (Sigma). Sections were counterstained with $1 \%$ methyl-green for $15 \mathrm{~min}$, dehydrated and mounted with Canadian balsam. For controls of the immunohistochemical staining, paraffin sections of the hearts of infected mice were processed with the same steps but without primary antibody. Sections of the hearts obtained from non-infected controls were submitted to all the steps for use as negative controls. Paraffin sections of the hearts of acutely infected mice that contained several parasite nests were processed for immunohistochemistry following the same steps above using a polyclonal anti-T. cruzi antibody.

Immunohistochemical identification of IDCs - Purified rat anti-mouse monoclonal antibodies for MAC-3, 33D1, CD11b (BD Pharmingen) and purified hamster anti-mouse CD11c (BD Pharmingen) were used. Sections of the hearts from the infected mice of both the untreated and treated groups and from non-infected controls were used. For immunolabeling of the IDCs in the heart, the purified rat anti-mouse monoclonal antibody for MAC3 (BD Biosciences Pharmigen) was applied to paraffin sections that were prepared as described above. After peroxidase blocking using $3 \% \mathrm{H}_{2} \mathrm{O}_{2}$ in methanol, the slides underwent antigen retrieval in a microwave oven using citrate buffer at $\mathrm{pH} 6$. The immunohistochemical procedures were the same as described above for the immunolabelling of $T$. cruzi antigens in paraffin sections. The MAC-3 monoclonal antibody was used at the dilution of 1:5 and incubated overnight in a humidified chamber at $4^{\circ} \mathrm{C}$. For immunolabelling with the purified rat anti-mouse monoclonal antibodies anti-CD11b (Mac$1 \alpha$-chain) and 33D1 (BD Biosciences Pharmigen) and with purified hamster anti-mouse CD11c monoclonal antibody, cryostat sections of the heart tissue cryopreserved into Tissue-Tek (OCT compound, Miles Inc, Diagnostic Division, Elkhart, USA) were used. The CD11b antibody was used at a dilution of 1:10. After being washed in PBS, the slides were treated with the secondary sheep anti-rat Ig-POD (peroxidase conjugated) Fab fragments (Boehringer Mannheim Biochemicals) at a dilution of 1:300. The sections treated with the primary antibody CD11c, a hamster anti-mouse antibody, were treated with a secondary goat anti-syrian hamster IgGPOD (Jackson Immuno-Research) at a dilution of 1:500 for $30 \mathrm{~min}$ at $37^{\circ} \mathrm{C}$. The complex was developed with $2.4 \% 3,3$ '-DAB and $1 \% \mathrm{H}_{2} \mathrm{O}_{2}$ at RT plus $1 \%$ dimethylsulfoxide (Sigma). Sections were counterstained with $1 \%$ methyl-green for $15 \mathrm{~min}$ and then dehydrated and mounted with Canadian balsam.

Quantitative evaluation of the number of IDCs presenting T. cruzi antigens immunolabelled with specific antibodies - Quantitative evaluation of IDCs immunolabelled for $T$. cruzi antigens was performed in the myocardium of the mice infected with either the Colombian strain or the 21SF-C3 clone. This evaluation was performed on mice from both points of sacrifice (150 and 200 days post-treatment) as follows: 1st group: infected with the Colombian strain, 150 days after treatment (16 mice, 7 untreated controls and 9 treated with benznidazole) and 200 days after treat- ment (13 mice, 7 untreated controls and 6 treated). 2nd group: infected with the 21SF-C3, 150 days after treatment $(15$ mice, 7 untreated controls and 8 treated) and 200 days after treatment (13 mice, 7 untreated controls and 6 treated).

The number of IDCs was evaluated in $5 \mu$ m-thick paraffin sections by counting 10 microscopic fields with Ocular 10X and Objective 40X, not successively, using a Zeiss Standard 18 microscope. The mean and standard deviation for the number of cells was established for each group and calculated for $1 \mathrm{~mm}^{2}$. Heart sections of non-infected controls were processed in the same way.

Morphometric evaluation of the number of inflammatory cells in the myocardium - For this analysis, the same cases that underwent quantification of IDCs were used. The degree of inflammation in the sections of the myocardium stained with H\&E was evaluated by examining a total area of $60 \mathrm{~mm}^{2}$ (5 fields of $12 \mathrm{~mm}^{2}$, not successively), using an ocular 10X and an objective 40X. For all semi-automated morphometry, an optical microscope (Zeiss) was used and the images were captured and evaluated using the program Axio Vision 3.1 (1998-2002), Carl Zeiss Vision GMbH (Zeppelinstrasse 4-85399 Munchen-Halbergnoss, Germany). The sectional areas of inflammation were directly measured and the total number of cells in the examined area was calculated.

Statistical analysis - Statistical analysis of the numbers of IDCs presenting T. cruzi antigens and infiltrated cells was performed with GraphPad Prism version 3.0 software. Kruskal-Wallis non-parametric and Dunnett's multiple comparative tests were considered significant when determinations reached $\mathrm{p}<0.05$.

\section{RESULTS}

\section{Mice chronically infected with the Colombian strain - results of treatment with benznidazole}

Cure rates - In the mice treated with benznidazole, $20.8 \%$ (5/24 mice) were cured.

Serology (IIFT) - Untreated mice presented titres of specific antibodies of 1:80-1.1280; the treated uncured mice presented titres from 1:20-1:1280 and the treated and cured mice had serological titres varying from 1:10-1:20.

Histopathological study - Fig. 1A represents a normal heart from one uninfected control mouse. In the infected, untreated controls (Table I), mild to moderate myocarditis was present in most of the cases; in a low percentage of cases, myocarditis was marked, with necrosis of cardiac cells and dense focal inflammatory infiltrates. Intracellular T. cruzi amastigotes were found in eight out of 16 cases (Fig. 1B). Both atrial and ventricular perivascular fibrosis were present. Skeletal muscle lesions were prominent in four of 16 cases, along with perivascular inflammation and arteritis, as well as necrosis and parasite debris in some myocells.

In mice treated with benznidazole, histopathology demonstrated a decrease in the intensity of myocardial inflammation and fibrosis in comparison to the untreated controls (Fig. 1C, D). Focal fragmentation of inter- 
stitial collagen matrix and the presence of intracellular amastigotes were seen in three out of 24 cases (Fig. 1C). Mild to moderate necro-inflammatory lesions were detected in skeletal muscles in two out of 24 cases. In two cases, moderate inflammatory perivascular infiltration was present (Table I).

Morphometric evaluation of the inflammatory infiltrates - In the myocardium of mice chronically infected with the Colombian strain and treated with benznidazole, a significant decrease in inflammatory cell number was observed in those sacrificed 150 days after the treatment. However, no significant differences were detected in the mice sacrificed 200 days after the end of treatment; this observation suggests that most of the regression of the inflammatory infiltration occurred in an earlier phase and is less apparent in the delayed phase (200 days) (Fig. 2A).
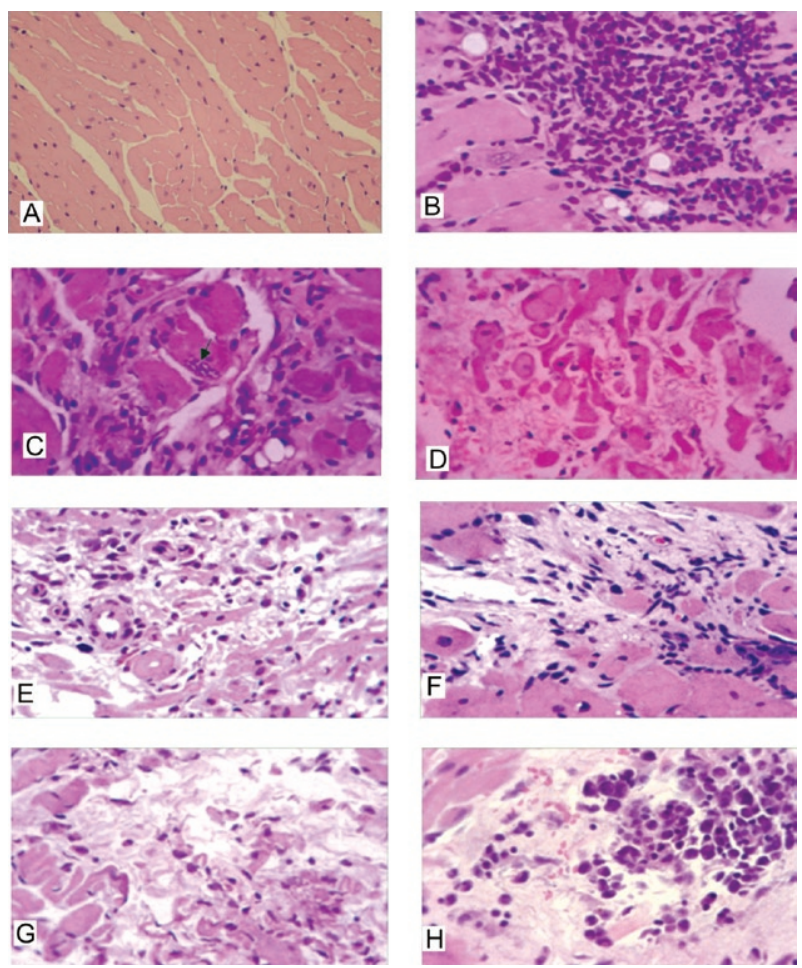

Fig. 1A: histological aspect of normal heart from uninfected control mouse; B-D: 1st group (Colombian strain). Histopathological aspects of the myocardium of mice chronically infected; B: untreated controls: moderate to intense interstitial, diffuse and focal mononuclear cells infiltrations with focal destruction of the myocytes. H\&E: 400X; C, D: treated with benznidazole: moderate to mild interstitial mononuclear infiltration and diffuse, loose, fragmented collagenic deposits. Intracellular amastigotes are present (arrow). H\&E: 400X; E-H: 2nd group (clone 21 SF-C3); E, F: untreated controls; E: mild interstitial mononuclear cells infiltration, presence of interstitial diffuse fibrillar matritial deposits; F: extensive area of cardiac cells destruction with collagen deposit and fibroblasts; G, H: treated with benznidazole; G: mild mononuclear diffuse infiltrate, focal destruction of myocytes and loose, fragmented collagenic deposit; H: area of myocytes destruction and focal infiltration with mononuclear cells showing picnosis, chromatin disintegration and apoptosis. H\&E: 400X.
TABLE I

Intensity of inflammatory lesions in mice infected with Trypanosoma cruzi (Colombian strain) ${ }^{a}$

\begin{tabular}{lccccc}
\hline & & \multicolumn{2}{c}{ Untreated } & \multicolumn{2}{c}{ Treated } \\
\cline { 3 - 6 } $\begin{array}{c}\text { Days post } \\
\text { treatment }\end{array}$ & $\begin{array}{c}\text { Degree } \\
\text { lesions }\end{array}$ & Heart & $\begin{array}{c}\text { Skeletal } \\
\text { muscle }\end{array}$ & Heart & $\begin{array}{c}\text { Skeletal } \\
\text { muscle }\end{array}$ \\
\hline 150 & - & $0 / 8$ & $0 / 8$ & $0 / 13$ & $2 / 13$ \\
& + & $0 / 8$ & $3 / 8$ & $9 / 13$ & $10 / 13$ \\
& ++ & $7 / 8$ & $4 / 8$ & $4 / 13$ & $1 / 13$ \\
& +++ & $1 / 8$ & $1 / 8$ & $0 / 13$ & $0 / 13$ \\
200 & - & $0 / 8$ & $0 / 8$ & $0 / 11$ & $4 / 11$ \\
& + & $1 / 8$ & $3 / 8$ & $8 / 11$ & $6 / 11$ \\
& ++ & $4 / 8$ & $2 / 8$ & $3 / 11$ & $1 / 11$ \\
& +++ & $3 / 8$ & $3 / 8$ & $0 / 11$ & $0 / 11$ \\
\hline
\end{tabular}

$a$ : treated with benznidazole and untreated controls; -: no lesions; +: mild focal lesions; ++: moderate focal and diffuse lesions; +++: intense inflammatory lesions.

\section{Mice infected with the clone 21SF-C3 (treated and untreated)}

Cure rates - Mice infected with the $21 \mathrm{SF}-\mathrm{C} 3$ and treated with benznidazole had a cure rate of $35.5 \%(12 / 33)$.

Serology - Untreated controls showed titres of specific antibodies varying from 1:160-1:1280, treated uncured mice showed titres from 1:10-1:320 and titres of antibodies in treated and cured mice varied from negative to 1:20.

Histopathology of infected untreated controls Chronic infection with clone 21SF-C3 caused mildto-moderate focal and diffuse myocarditis with focal myocardial necrosis (Fig. 1E, F). Atrial and ventricular interstitial fibrosis was also present. No cases with intense lesions were detected (Table II).

Mice treated with benznidazole - Inflammatory lesions in the myocardium were mild to moderate, with focal necrosis of myocytes (Fig. 1G). Focal inflammatory infiltrates revealed the presence of mononuclear cells in apoptosis (Fig. 1H). Mild-to-moderate interstitial fibrosis, with a loose aspect and fragmentation of collagen fibres, was present. Only one case showed intense lesions (Table II).

Morphometric evaluation of the inflammatory infiltrates in the hearts of mice chronically infected with strain 21SF-C3, untreated or treated with benznidazole There was no difference in inflammatory infiltrates between treated and untreated mice examined 150 or 200 days after treatment (Fig. 2B).

\section{Evaluation of IDCs}

Controls - Monoclonal antibodies: anti-MAC-3, 33D1, CD11b and CD11c (BD Bioscience Pharmigen) immunolabelled dendritic cells of the heart in both 
TABLE II

Intensity of inflammatory lesions in mice infected with Trypanosoma cruzi (clone 21SF-C3) ${ }^{a}$

\begin{tabular}{lccccc}
\hline & & \multicolumn{2}{c}{ Untreated } & \multicolumn{2}{c}{ Treated } \\
\cline { 3 - 6 } $\begin{array}{c}\text { Days post } \\
\text { treatment }\end{array}$ & $\begin{array}{c}\text { Degree } \\
\text { lesions }\end{array}$ & Heart & $\begin{array}{c}\text { Skeletal } \\
\text { muscle }\end{array}$ & Heart & $\begin{array}{c}\text { Skeletal } \\
\text { muscle }\end{array}$ \\
\hline 150 & - & $0 / 14$ & $6 / 14$ & $0 / 17$ & $5 / 16$ \\
& + & $7 / 14$ & $8 / 14$ & $11 / 17$ & $10 / 16$ \\
& ++ & $7 / 14$ & $0 / 14$ & $6 / 17$ & $0 / 16$ \\
200 & +++ & $0 / 14$ & $0 / 14$ & $0 / 17$ & $0 / 16$ \\
& - & $0 / 10$ & $3 / 10$ & $0 / 17$ & $14 / 17$ \\
& + & $3 / 10$ & $4 / 10$ & $10 / 17$ & $2 / 17$ \\
& ++ & $7 / 10$ & $3 / 10$ & $6 / 17$ & $1 / 17$ \\
& +++ & $0 / 10$ & $0 / 10$ & $1 / 17$ & $0 / 17$ \\
\hline
\end{tabular}

$a$ : treated with benznidazole and untreated controls; -: no lesions; +: mild focal lesions; ++: moderate focal and diffuse lesions; +++: intense inflammatory lesions.

infected and non-infected controls within the interstitium of the heart, adherent to the myocardiocyte basal membrane (Fig. 3).

Immunolabelling of T. cruzi antigens in the IDCs The dendritic cells were detected in the myocardium in all cases, showing positive immunostaining for T. cruzi antigens both in mice infected with the Colombian strain (Fig. 4A-C) and with the clone 21SF-C3 (Fig. 4D-F). Antigens appeared as granular deposits in the membrane of isolated cells or in groups of 2-3 of these cells. They were also present within inflammatory foci, in lymphocytes and in macrophages associated with disintegrated myocells. Amastigotes of $T$. cruzi were rarely detected in the cytoplasm of IDCs, as shown in Fig. 4F.

\section{Quantitative evaluation of IDCs}

1st group - Colombian strain - The number of IDCs per $\mathrm{mm}^{2}$ is shown in Fig. 5A. A significant decrease in the number of IDCs was detected in treated mice sacrificed 200 days after the end of treatment. A decrease was also observed in those sacrificed at 150 days, although without statistical significance.

2nd group - clone 21 SF-C3 - The number of IDCs per $\mathrm{mm}^{2}$ is shown in Fig. 5B. A significant decrease in the number of IDCs was detected in treated mice in comparison with the untreated controls in those sacrificed 150 days after the end of treatment. A decrease was also observed in those sacrificed at 200 days, although without significance when compared with untreated controls.

Immunohistochemical reactions using the anti-T. cruzi polyclonal antibody in hearts of mice from the two experimental groups - Positive immunostaining revealed the presence of $T$. cruzi intracardiomyocytic amastigotes in infected mice of both groups, both in untreated controls and in those treated with benznida-
A

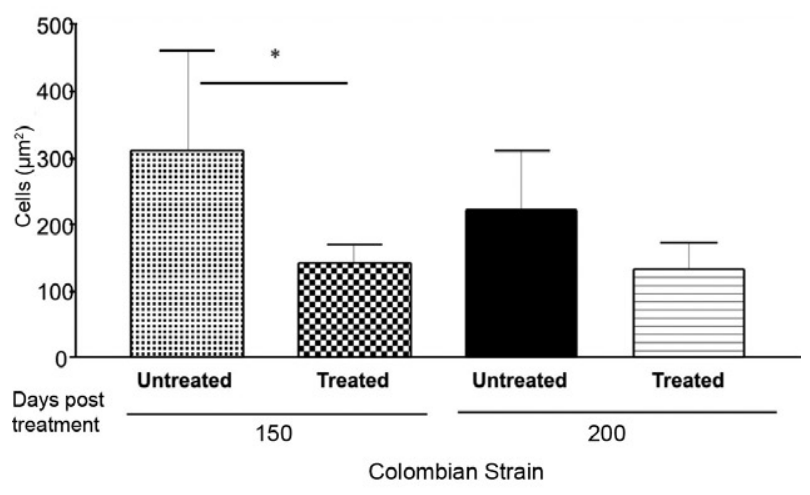

B

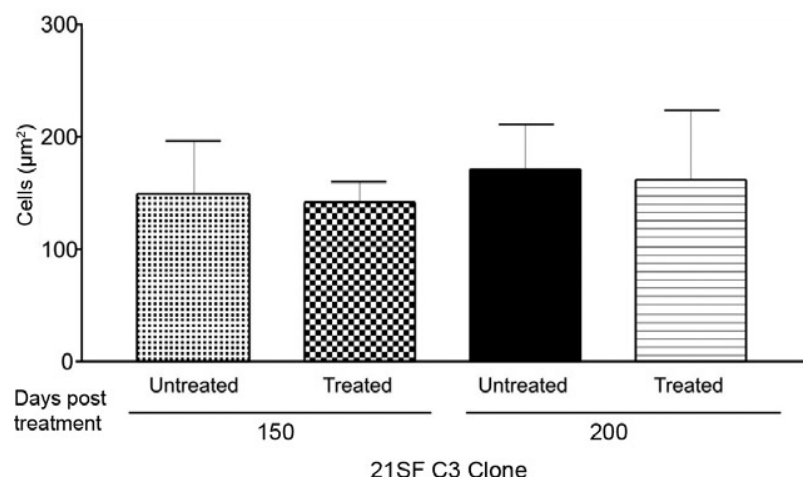

Fig. 2A, B: morphometric evaluation of the number of mononuclear inflammatory cells in the myocardium of mice infected with two different strains of Trypanosoma cruzi, treated and untreated with bennidazole; A: Colombian strain: significant decrease of inflammatory cells was detected in the heart of the treated mice sacrificed 150 days after treatment. A decrease of inflammatory cells both in untreated and treated group was observed 200 days after treatment, but without statistical significance; B: clone 21SF-C3: no significant difference has been detected between the treated and untreated mice either at 150 or 200 days post-treatment. Statististical analysis $(*)$ : non-parametric test - Kruskal-Wallis $\mathrm{p}<0.05$.

zole but not cured. Parasite debris was related to disintegrated parasitised myocells and was also seen within inflammatory foci.

\section{DISCUSSION}

Treatment of mice chronically infected with $T$. cru$z i$ with benznidazole resulted in a marked decrease in presence of myocardial necrotic-inflammatory lesions and fibrosis. However, a residual myocarditis remained even in animals in which parasitological tests were negative (i.e., those that were cured). An investigation into the importance of antigenic stimulation by parasite antigens sequestered within heart IDCs has been performed in treated and untreated mice. The trypanocidal activity of the drug is probably responsible for the total or partial elimination of the parasites, thus leading toward a regression of the inflammatory reaction. As suggested in previous studies (Andrade et al. 1989a, b, 

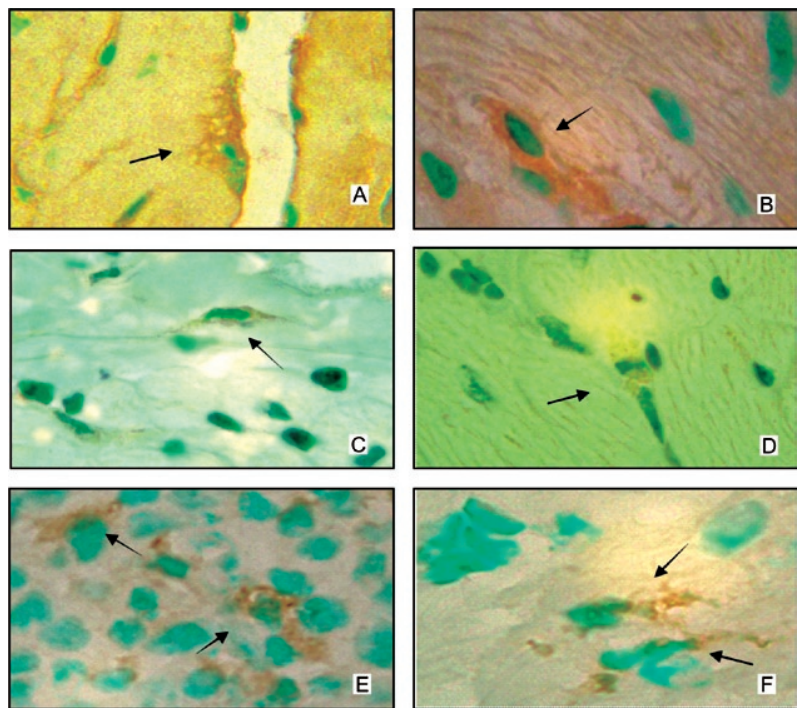

Fig. 3: immunohistochemistry. Interstitial dendritic cells (IDCs) of the heart were immunolabeled either with anti-MAC-3, anti-33D1, anti$\mathrm{CD} 11 \mathrm{~b}$ and anti-CD11c monoclonal antibodies. A: dendritic cell of the heart with many processes, immunolabeled with anti-MAC-3; B: IDC immunolabeled with the 33D1 monoclonal antibody; C: IDC immunolabeled with the anti-CD11b monoclonal antibody; D-F: presence of the IDCs immunolabeled with anti-CD11c monoclonal antibody.
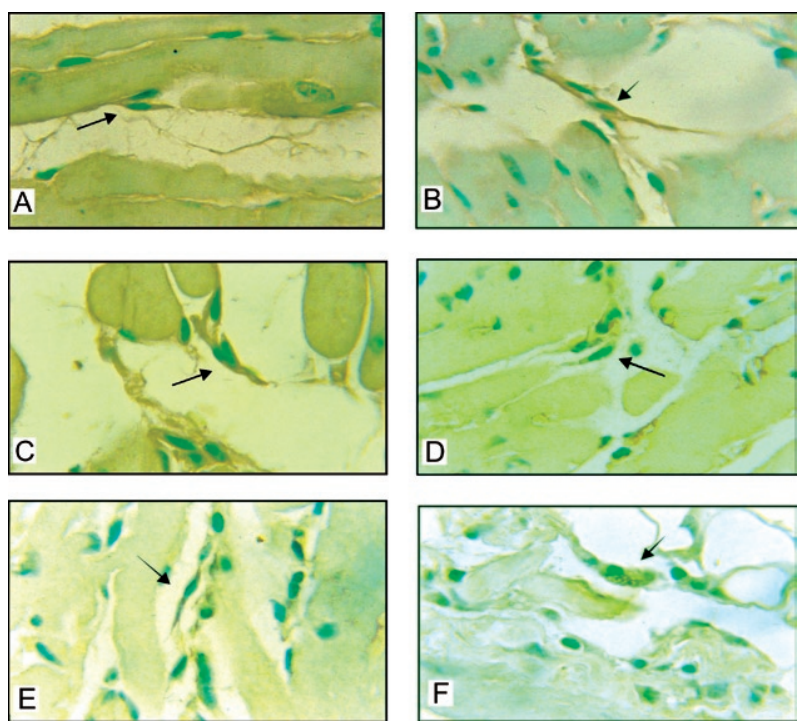

Fig. 4: immunohistochemistry-anti-Trypanosoma cruzi polyclonal antibody. The dendritic cells (arrows) are elongated with fine cytoplasmic processes, scanty cytoplasm and central, pale, round nucleus immunolabeled with anti-T. cruzi antibodies. A-C: Colombian strain; D-F: clone 21 SF-C3; F: IDC with abundant cytoplasm and containing T. cruzi amastigotes. 1.000X.

1991b), regression of the tissue lesions after treatment indicates the importance of the parasite and its antigens in the pathogenesis of chronic T. cruzi myocarditis. Thus, the presence of residual inflammation could be related to persisting antigenic stimulation from the
A

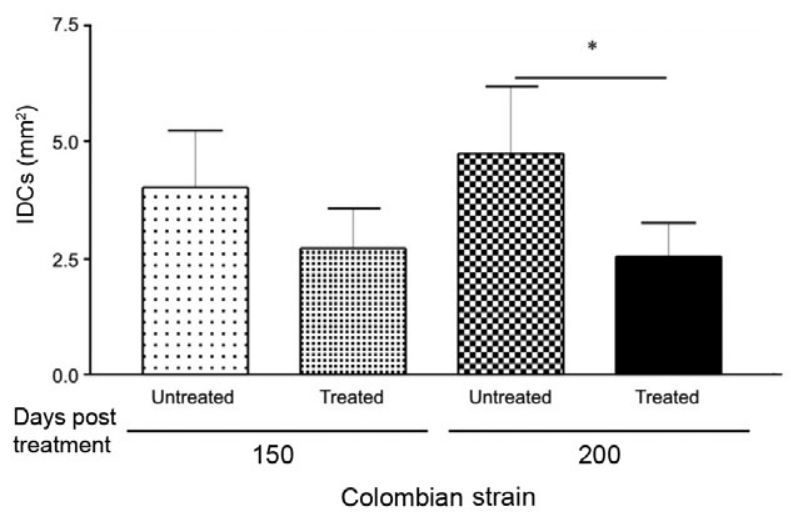

B

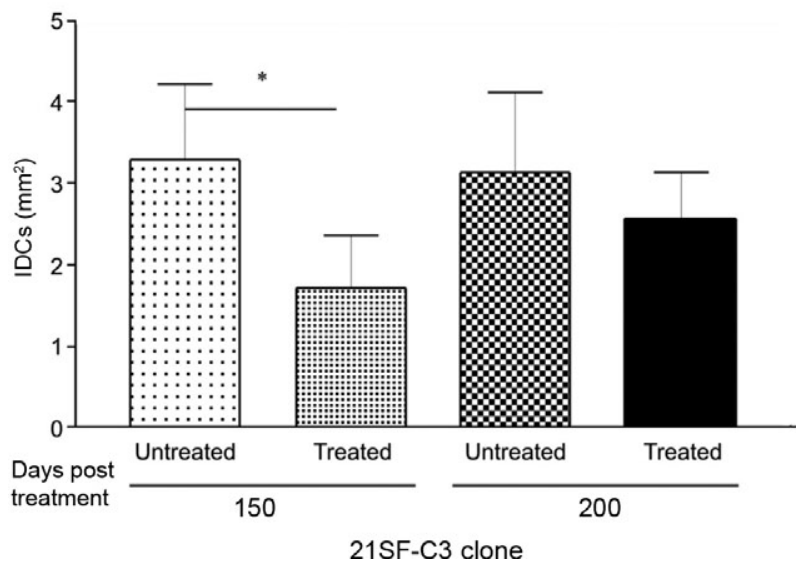

Fig. 5: quantitative evaluation of the number of interstitial dendritic cells (IDCs) immunolabelled for Trypanosoma cruzi antigens in the myocardium of mice chronically infected with different strains of $T$. cruzi untreated and treated with benznidazole. A: Colombian strain: there is a significant decrease in the number of dendritic cells in the myocardium of the mice sacrificed 200 days after treatment. In those sacrificed 150 days after treatment, there is also a decrease on the number of cells, but without statistical significance; B: clone 21 SFC3: a significant decrease in the number of IDCs has been observed in the treated mice, 150 days after treatment. In the mice sacrificed 200 days after the end of treatment, a decrease of the IDCs was also observed although without significance. Statististical analysis $(*)$ : non-parametric test - Kruskal-Wallis $\mathrm{p}<0.05$.

T-cell immunological compartment. Several data are in agreement with the detection of $T$. cruzi antigens in the myocardium, including: (i) the presence of intracellular amastigotes (in uncured mice), (ii) the presence of antigenic debris left over from parasite destruction (Higuchi et al. 1993, Jones et al. 1993) and (iii) the presence of antigens sequestered on the membrane of IDCs (Andrade et al. 2000).

The IDCs are characterised as lymphoid dendritic cells that specifically migrate to the T-cell zone of the spleen. Demonstration of parasite antigens on the membrane of IDCs suggests that these cells are 
involved in the pathogenesis of chronic myocarditis. According to Flores-Romo (2001), dendritic cells trap, engulf and digest antigens from diverse origins (antigen processing); antigenic fragments are then regurgitated and exposed at the cell surface through three molecular pathways: MHC-I, MHC-II and CD1. These cells concomitantly develop co-stimulatory molecules, such as CD40, CD80 and CD86, along with the potential to produce cytokines such as IL-12 and other chemokines. Specific cytokines up-regulate both the presentation and sensitisation functions of the accessory cells which require the action of IL-1, a dendritic cell activator (Steinman 1988). Dendritic cells mediate Th-1 development and IFN $\gamma$ production (Heufler et al. 1996). These cells reside in the tissues as an immature stage and respond to various chemoattractants from inflammation sites. They can be activated by microorganisms and inflammatory stimuli, undergo a maturation process and become potent stimulators of $\mathrm{T}$ cells (Hollemweguer et al. 2001).

In previous studies, Andrade et al. (2000) demonstrated a connection between DC numbers and inflammation intensity in the hearts of infected dogs. In the myocardium of dogs at different phases of infection, IDCs expressed T. cruzi antigens in their membrane, as evidenced by immunohistochemical staining. Quantitative evaluation of the number of dendritic cells in canine hearts in acute and chronic infections has demonstrated that the number of IDCs is proportional to the intensity of the inflammatory infiltration. IDC number is significantly higher in acute and chronic myocarditis, as compared to the findings seen in the indeterminate form of the disease.

T. cruzi antigens have been previously found in the membranes of follicular dendritic cells of the spleen (Andrade et al. 1991a) in chronically infected mice. These cells are present in the germinal centres of lymphoid follicles of the spleen; they are important antigen-presenting cells to memory B-lymphocytes and they are involved with the humoral response to $T$. cruzi infection.

Other studies on T. cruzi participation in the behaviour of lymphoid DCs that are responsible for T-cell sensitisation have been conducted in vitro using spleen cells, which are not correlated to IDCs. According to Chaussabel et al. (2003), there is a huge increase in the number of CD11c-postive DCs in the spleen during the acute phase of $T$. cruzi infection (14-21 days post infection), with T-cell depletion seen during the course of infection. Soto et al. (2003) have also studied the behaviour of splenic dendritic cells and their relationship with $T$. cruzi infection, showing $T$ cell stimulatory activity of dendritic cells. In the present study our interest was in the response of the IDCs to prolonged chronic experimental infection of mice with either of two different strains of $T$. cruzi and the effects of treatment with benznidazole. A direct effect of benznidazole on the regression of lesions in the early acute phase of the infection has been shown by several authors. Olivieri et al. (2006) showed that benznidazole treatment during the acute phase of infection inhibits the innate response to T. cruzi. Lima et al. (2001) showed an in situ decreased expression of TNF- $\alpha$ in necrotic areas of the spleen during acute infection with a macrophagotropic strain of $T$. cruzi after treatment with benznidazole. However, in the present investigation, a direct effect of benznidazole on the regression of inflammatory infiltrates is not probable because the animals were treated in the chronic phase of infection (90 and 120 days), when the cellular immunological response is predominant. Furthermore, the animals were sacrificed in the late phase (150 and 200 days post-treatment), which is sufficient time for the elimination of the drug from the animal.

The findings of the present investigation indicate that elimination of parasites was related to a decrease in the number of dendritic cells in the hearts of chronically infected mice treated with benznidazole. The reduction of IDCs was significant at 200 days post-treatment of the infection with the Colombian strain and 150 days after treatment of the infection with clone 21SF-C3, revealing a difference in the modulation of dendritic cells related to the parasite strain. The significant decrease in the number of inflammatory cells was also more evident in animals chronically infected with the Colombian strain. This was not observed with the clone 21SF-C3, although a clear regression of focal myocardial necrosis and interstitial fibrosis was observed. Probably, the presence of apoptotic nuclei seen in the hearts of mice treated with benznidazole interfered in the morphometric analysis. These findings indicate that the histopathological and morphometric combined evaluation is important. The Colombian strain, which is Biodeme Type III (T. cruzi I) and clone 21SF-C3, which is Biodeme type II (T. cruzi II), differ in several biological characteristics, including their susceptibility to antiparasitic therapy. The two strains differed in their pathogenicity, with the Colombian strain being more pathogenic, determined by moreintense histopathological lesions (Andrade 1990). The 21SF-C3 clone, derived from the Type II strain 21SF, is less pathogenic and demonstrated mild to moderate lesions in chronic infection. A difference in the modulation of MHC-II in splenic dendritic cells by T. cruzi strains with different degrees of virulence has been shown by Soto et al. (2003). A decrease in the number of dendritic cells and a regression of chronic myocarditis were detected in treated mice. Residual inflammatory infiltrates were present and correlated with the presence of dendritic cells carrying parasite antigens, although in lower number, in comparison to the untreated mice. This raises the possibility that regression of the cellular immune response after specific anti-T. cruzi therapy that leads to a cure may also depend on the clearance of parasite antigens from heart dendritic cells.

The time for clearance of T. cruzi antigens from the lymphoid dendritic cells in mice has not been determined; however, antigens were detected until the end of the present investigation, that is, 200 days after treatment. The follicular dendritic cells, responsible for B cell sensitisation, carried parasite antigens up to six months after treatment and cure, being not investigated after that period (Andrade et al. 1991a). The persistence of parasite antigens within splenic follicular dendritic cells explains the maintenance of positive serological 
results for several months following treatment and cure. Thus, treatment with specific anti-T. cruzi drugs is important not only for eliminating living parasites, but also for diminishing stimulation by IDC presentation of parasite antigens.

\section{REFERENCES}

Andrade SG 1990. Influence of Trypanosoma cruzi strain on the pathogenesis of chronic myocardiopathy in mice. Mem Inst Oswaldo Cruz 85: 17-27.

Andrade SG, Freitas LAR, Peyrol S, Pimentel AR, Sadigursky M 1991a. Experimental chemotherapy of Trypanosoma cruzi infection: persistence of parasite antigens and positive serology in parasitologically cured mice. Bull World Health Organ 69: 191-197.

Andrade SG, Magalhães JB 1997. Biodemes and zymodemes of Trypanosoma cruzi strains: correlations with clinical data and experimental pathology. Rev Soc Bras Med Trop 30: 27-35.

Andrade SG, Magalhães JB, Pontes AL 1985. Evaluation of chemotherapy with benzonidazole and nifurtimox in mice infected with Trypanosoma cruzi strains of different types. Bull World Health Organ 63: 721-726.

Andrade SG, Magalhães JB, Pontes AL 1989a. Terapêutica da fase crônica da infecção experimental pelo Trypanosoma cruzi com o benzonidazol e o nifurtimox. Rev Soc Bras Med Trop 22: $113-118$

Andrade SG, Pimentel AR, Andrade ZA 2000. Interstitial dendritic cells of the heart harbor Trypanosoma cruzi antigens in experimentally infected dogs: importance for the pathogenesis of chagasic myocarditis. Am J Trop Med Hyg 63: 64-70.

Andrade SG, Silva RC, Santiago CM 1989b. Treatment of chronic experimental Trypanosoma cruzi infections in mice with MK436, a 2-substituted 5-nitroimidazole. Bull World Health Organ 67: 509-514.

Andrade SG, Stocker-Guerret S, Pimentel AS, Grimaud JA 1991b. Reversibility of cardiac fibrosis in mice chronically infected with Trypanosoma cruzi under specific chemotherapy. Mem Inst Oswaldo Cruz 86: 187-200.

Andrade ZA 1991. Pathogenesis of Chagas disease. Res Imunol 42: $126-129$.

Andrade ZA 1999. Immunopathology of Chagas disease. Mem Inst Oswaldo Cruz 94: 71-80.

Anonymous 1999. Recommendations from a Satellite Meeting. Mem Inst Oswaldo Cruz 94: 29-432.

Campos RF, Guerreiro ML, Sobral KSC, Lima, RDEC, Andrade SG 2005. Response to chemotherapy with benznidazole of clones isolated from the 21SF strain of Trypanosoma cruzi (biodeme Type II, Trypanosoma cruzi. II. Rev Soc Bras Med Trop 38: 142-146.

Chaussabel D, Pajak B, Vercruysse V 2003. Alteration of migration and maturation of dendritic cells and T-cell depletion in the course of experimental Trypanosoma cruzi infection. Lab Invest 83: $1373-1382$

Cunha-Neto E, Coelho V, Guilherme L, Fiorelli A, Stolf N, Kalil J 1996. Autoimmunity in Chagas' disease. Identification of cardiac myosin-B13 Trypanosoma cruzi protein cross-reactive $\mathrm{T}$ cell clones in heart lesions of a chronic Chagas' cardiomyopathy patient. J Clin Invest 98: 1709-1712.

Cunha-Neto E, Duranti M, Gruber A, Zingales B, DE Messias I, Stolf N, Bellotti G, Patarroyo ME, Pilleggi F, Kalil J 1995. Autoimmunity in Chagas disease cardiopathy: biological relevance of a cardiac myosin-specific epitope crossreactive to an immu- nodominant Trypanosoma cruzi antigen. Proc Natl Acad Sci 92: 3541-3545.

Dos Reis GA 1997. Cell-mediated immunity in experimental Trypanosoma cruzi infection. Parasitol Today 13: 335-342.

Drakesmith H, Chain B, Beverley P 2000. How can dendritic cells cause autoimmune disease? Immunol Today 21: 214-217.

Flores-Romo L 2001. In vivo maturation and migration of dendritic cells. Immunology 102: 255-262.

Girones N, Fresno M 2003. Etiology of Chagas disease myocarditis: autoimmunity, parasite persistence, or both? Trends Parasitol 19: 19-22.

Hart DN, Fabre JW 1981. Demonstration and characterization of Iapositive dendritic cells in the interstitial connective tissues of rat heart and other tissues, but not brain. J Exp Med 154: 347-361.

Hart DN, Mckenzie JL 1990. Interstitial dendritic cells. Int Rev Immunol 6: 127-138.

Heufler C, Koch F, Stanzl U, Topar G, Wysocka M, Trinchieri G, Enl A, Steiman RM, Romani N, Schuler G 1996. Interleukin-12 is produced by dendritic cells and mediates $\mathrm{T}$ helper 1 development as well as interferon-gamma production by $\mathrm{T}$ helper 1 cells. Eur J Immunol 26: 659-668.

Higuchi ML, Brito T, Reis MM, Barbosa A, Bellotti G, Pereira-Barreto AC, Pileggi F 1993. Correlation between Trypanosoma cruzi parasitism and myocarditis: light microscopy and immunohistochemical findings. Cardiovasc Pathol 2: 101-106.

Hollemweguer EJ, Koppelman B, Dong J 2001. Dendritic cells and antigen processing and presentation. Hotlines 6: 14-21.

Inaba K, Young JW, Steinman RM 1987. Direct activation of CD8 ${ }^{+}$cytotoxic T lymphocytes by dendritic cells. J Exp Med 166: 182-194.

Jones EM, Colley DG, Tostes S, Lopes ER, Vnencak-Jones CL, Mccurley TL 1993. Amplification of a Trypanosoma cruzi DNA sequence from inflammatory lesions human chagasic cardiomyopathy. Am J Trop Med Hyg 48: 348-357.

Lima ES, Andrade ZA, Andrade SG 2001. TNFa is expressed at sites of parasite and tissue destruction in the spleen of mice acutely infected with Trypanosoma cruzi. Int J Exp Pathol 82: 327-336.

Olivieri BP, Souza AP, Cotta-de-Almeida V, Castro SL, Araújo-Jorge T 2006. Trypanosoma cruzi: alteration in the lymphoid compartments following interruption of infection by early acute benznidazole therapy in mice. Exper Parasitol 114: 228-234.

Soto ACD, Mirkin GA, Solana ME, Cappa SMG 2003. Trypanosoma cruzi infection modulates in vivo expression of major histocompatibility complex class II molecules on antigen-presenting cells and T-cell stimulatory activity of dendritic cells in a strain-dependent manner. Infect Immun 71: 1194-1199.

Steinman RM 1988. Cytokines amplify the function of accessory cells. Immunol Lett 17: 197-202.

Steinman RM 1991. The dendritic cell system and its role in immunogenicity. Annu Rev Immunol 9: 271-296.

Tarleton RL 1995. The role of T cells in Trypanosoma cruzi infections. Parasitol Today 11: 7-9.

Tarleton RL 2001. Parasite persistence in the aetiology of Chagas disease. Int J Parasitol 31: 550-554.

Tarleton RL, Grusby MJ, Postan M, Glimcher LH 1996. Trypanosoma cruzi infection in MHC-deficient mice: further evidence for the role of both class I and class II-restricted T cells in immune resistance and disease. Int Immunol 8: 13-22. 